\title{
Two kinds of Hilbert-type integral inequalities in the whole plane
}

\author{
Bicheng Yang ${ }^{1 *}$ and Qiang Chen ${ }^{2}$
}

\author{
"Correspondence: \\ bcyang@gdei.edu.cn; \\ bcyang818@163.com \\ 1 Department of Mathematics, \\ Guangdong University of \\ Education, Guangzhou, Guangdong \\ 510303, P.R. China \\ Full list of author information is \\ available at the end of the article
}

\begin{abstract}
By the way of using real analysis and estimating the weight functions, two kinds of Hilbert-type integral inequalities in the whole plane with a non-homogeneous kernel and a homogeneous kernel are given. The constant factor related to the triangle functions is proved to be the best possible. We also consider the equivalent forms, the reverses, some particular cases, and two kinds of operator expressions.
\end{abstract}

MSC: $26 \mathrm{D} 15$

Keywords: Hilbert-type integral inequality; weight function; equivalent form; reverse; operator expression

\section{Introduction}

Assuming that $f(x), g(y) \geq 0,0<\int_{0}^{\infty} f^{2}(x) d x<\infty$ and $0<\int_{0}^{\infty} g^{2}(y) d y<\infty$, we have the following Hilbert integral inequality $(c f .[1])$ :

$$
\int_{0}^{\infty} \int_{0}^{\infty} \frac{f(x) g(y)}{x+y} d x d y<\pi\left(\int_{0}^{\infty} f^{2}(x) d x \int_{0}^{\infty} g^{2}(y) d y\right)^{\frac{1}{2}}
$$

where the constant factor $\pi$ is the best possible. Inequality (1) together with the discrete form is important in analysis and its applications $(c f .[1,2])$. In recent years, applying weight functions and introducing parameters, many extensions of (1) were given by Yang ( $c f$. [3]). Noticing that inequality (1) is a homogeneous kernel of degree -1 , in 2009, a survey of the study of Hilbert-type inequalities with the homogeneous kernels of negative number degrees was given by [4]. Recently, some inequalities with the homogeneous kernels of degree 0 and non-homogeneous kernels were studied ( $c f$. [5-10]). The other kinds of Hilbert-type inequalities were provided by [11-15]. All of the above integral inequalities are built in the quarter plane of the first quadrant.

In 2007, Yang [16] first gave a Hilbert-type integral inequality in the whole plane as follows:

$$
\begin{aligned}
& \int_{-\infty}^{\infty} \int_{-\infty}^{\infty} \frac{f(x) g(y)}{\left(1+e^{x+y}\right)^{\lambda}} d x d y \\
& \quad<B\left(\frac{\lambda}{2}, \frac{\lambda}{2}\right)\left(\int_{-\infty}^{\infty} e^{-\lambda x} f^{2}(x) d x \int_{-\infty}^{\infty} e^{-\lambda y} g^{2}(y) d y\right)^{\frac{1}{2}}
\end{aligned}
$$

๑ 2015 Yang and Chen; licensee Springer. This is an Open Access article distributed under the terms of the Creative Commons Attribution License (http://creativecommons.org/licenses/by/4.0), which permits unrestricted use, distribution, and reproduction in any medium, provided the original work is properly credited. 
where the constant factor $B\left(\frac{\lambda}{2}, \frac{\lambda}{2}\right)(\lambda>0)$ is the best possible, and $B(u, v)(u, v>0)$ is the beta function (cf. [17]). He et al. [18-28] also provided some Hilbert-type integral inequalities in the whole plane.

In this paper, by the way of using real analysis and estimating the weight functions, two kinds of Hilbert-type integral inequalities in the whole plane with a non-homogeneous kernel and a homogeneous kernel are given. The constant factor related to the triangle functions is proved to be the best possible. We also consider the equivalent forms, the reverses, some particular cases, and two kinds of operator expressions.

\section{Some lemmas}

In the following, we use the following formula (cf. [1]):

$$
\int_{0}^{\infty} \frac{(\ln t) t^{a-1}}{t-1} d t=[B(1-a, a)]^{2}=\left[\frac{\pi}{\sin a \pi}\right]^{2} \quad(0<a<1) .
$$

Lemma 1 If $0<\alpha_{1} \leq \alpha_{2}<\pi, \mu, \sigma>0, \mu+\sigma=\lambda, \gamma \in\left\{a ; a=\frac{1}{2 k+1}, 2 k-1(k \in \mathbf{N}=\{1,2, \ldots\})\right\}$, $\delta \in\{-1,1\}$, we define two weight functions $\omega(\sigma, y)$ and $\varpi(\sigma, x)(y, x \in \mathbf{R}=(-\infty, \infty))$ as follows:

$$
\begin{aligned}
& \omega(\sigma, y):=\int_{-\infty}^{\infty} \max _{i \in\{1,2\}} \frac{\ln \left[\left|x^{\delta} y\right|^{\gamma}+\left(x^{\delta} y\right)^{\gamma} \cos \alpha_{i}\right]}{\left[\left|x^{\delta} y\right|^{\gamma}+\left(x^{\delta} y\right)^{\gamma} \cos \alpha_{i}\right]^{\lambda / \gamma}-1} \frac{|y|^{\sigma}}{|x|^{1-\delta \sigma}} d x, \\
& \varpi(\sigma, x):=\int_{-\infty}^{\infty} \max _{i \in\{1,2\}} \frac{\ln \left[\left|x^{\delta} y\right|^{\gamma}+\left(x^{\delta} y\right)^{\gamma} \cos \alpha_{i}\right]}{\left[\left|x^{\delta} y\right|^{\gamma}+\left(x^{\delta} y\right)^{\gamma} \cos \alpha_{i}\right]^{\lambda / \gamma}-1} \frac{|x|^{\delta \sigma}}{|y|^{1-\sigma}} d y .
\end{aligned}
$$

Then for $y, x \in \mathbf{R} \backslash\{0\}$, we have

$$
\begin{aligned}
& \omega(\sigma, y)=\varpi(\sigma, x)=K(\sigma), \\
& K(\sigma):=\frac{\gamma}{2^{\frac{\sigma}{\gamma}}}\left[\left(\sec \frac{\alpha_{2}}{2}\right)^{\frac{2 \sigma}{\gamma}}+\left(\csc \frac{\alpha_{1}}{2}\right)^{\frac{2 \sigma}{\gamma}}\right]\left[\frac{\pi}{\lambda \sin \left(\frac{\pi \sigma}{\lambda}\right)}\right]^{2} \in \mathbf{R}_{+} .
\end{aligned}
$$

Proof Setting $u=x^{\delta} y$ in (4), for $y \in \mathbf{R} \backslash\{0\}$, we find $x=y^{\frac{-1}{\delta}} u^{\frac{1}{\delta}}, d x=\frac{1}{\delta} y^{\frac{-1}{\delta}} u^{\frac{1}{\delta}-1} d u$, and

$$
\begin{aligned}
\omega(\sigma, y)= & \left|\frac{1}{\delta}\right| \int_{-\infty}^{\infty} \max _{i \in\{1,2\}} \frac{\ln \left(|u|^{\gamma}+u^{\gamma} \cos \alpha_{i}\right)}{\left(|u|^{\gamma}+u^{\gamma} \cos \alpha_{i}\right)^{\lambda / \gamma}-1}|u|^{\sigma-1} d u \\
= & \int_{0}^{\infty} \max _{i \in\{1,2\}} \frac{\ln \left[u^{\gamma}\left(1+\cos \alpha_{i}\right)\right]}{u^{\lambda}\left(1+\cos \alpha_{i}\right)^{\lambda / \gamma}-1} u^{\sigma-1} d u \\
& +\int_{-\infty}^{0} \max _{i \in\{1,2\}} \frac{\ln \left[(-u)^{\gamma}\left(1-\cos \alpha_{i}\right)\right]}{(-u)^{\lambda}\left(1-\cos \alpha_{i}\right)^{\lambda / \gamma}-1}(-u)^{\sigma-1} d u \\
= & \gamma\left\{\int_{0}^{\infty} \max _{i \in\{1,2\}} \frac{\ln \left[u\left(1+\cos \alpha_{i}\right)^{1 / \gamma}\right]}{\left[u\left(1+\cos \alpha_{i}\right)^{1 / \gamma}\right]^{\lambda}-1} u^{\sigma-1} d u\right. \\
& \left.+\int_{0}^{\infty} \max _{i \in\{1,2\}} \frac{\ln \left[u\left(1-\cos \alpha_{i}\right)^{1 / \gamma}\right]}{\left[u\left(1-\cos \alpha_{i}\right)^{1 / \gamma}\right]^{\lambda}-1} u^{\sigma-1} d u\right\} .
\end{aligned}
$$


In view of the function $\frac{\ln t}{t^{\lambda}-1}$ being strictly decreasing in $\mathbf{R}_{+}(c f$. [3]), we have

$$
\begin{aligned}
\omega(\sigma, y)= & \int_{0}^{\infty} \frac{\ln \left[u^{\gamma}\left(1+\cos \alpha_{2}\right)\right]}{\left[u\left(1+\cos \alpha_{2}\right)^{1 / \gamma}\right]^{\lambda}-1} u^{\sigma-1} d u \\
& +\int_{0}^{\infty} \frac{\ln \left[u^{\gamma}\left(1-\cos \alpha_{1}\right)\right]}{\left[u\left(1-\cos \alpha_{1}\right)^{1 / \gamma}\right]^{\lambda}-1} u^{\sigma-1} d u
\end{aligned}
$$

Setting $t=\left[u\left(1+\cos \alpha_{2}\right)^{1 / \gamma}\right]^{\lambda}\left(t=\left[u\left(1-\cos \alpha_{1}\right)^{1 / \gamma}\right]^{\lambda}\right)$ in the above first (second) integral, by calculations and (3), it follows that

$$
\omega(\sigma, y)=\frac{\gamma}{\lambda^{2}}\left[\left(\frac{\sec ^{2} \frac{\alpha_{2}}{2}}{2}\right)^{\frac{\sigma}{\gamma}}+\left(\frac{\csc ^{2} \frac{\alpha_{1}}{2}}{2}\right)^{\frac{\sigma}{\gamma}}\right] \int_{0}^{\infty} \frac{\ln t}{t-1} t^{\frac{\sigma}{\lambda}-1} d t=K(\sigma)
$$

Setting $u=x^{\delta} y$ in (5), for $x \in \mathbf{R} \backslash\{0\}$, we find $y=x^{-\delta} u, d y=x^{-\delta} d u$ and

$$
\varpi(\sigma, x)=\int_{-\infty}^{\infty} \max _{i \in\{1,2\}} \frac{\ln \left(|u|^{\gamma}+u^{\gamma} \cos \alpha_{i}\right)|u|^{\sigma-1}}{\left(|u|^{\gamma}+u^{\gamma} \cos \alpha_{i}\right)^{\lambda / \gamma}-1} d u=K(\sigma)
$$

Hence we have (6).

Remark 1 If we replace $\max _{i \in\{1,2\}}$ by $\min _{i \in\{1,2\}}$ in (4) and (5), then we must exchange $\alpha_{1}$ and $\alpha_{2}$ in (6).

Lemma 2 If $p>1, \frac{1}{p}+\frac{1}{q}=1,0<\alpha_{1} \leq \alpha_{2}<\pi, \mu, \sigma>0, \mu+\sigma=\lambda, \gamma \in\left\{a ; a=\frac{1}{2 k+1}, 2 k-1(k \in\right.$ $\mathbf{N})\}, \delta \in\{-1,1\}, K(\sigma)$ is indicated by $(6), f(x)$ is a non-negative measurable function in $\mathbf{R}$, then we have

$$
\begin{aligned}
J & :=\int_{-\infty}^{\infty}|y|^{p \sigma-1}\left\{\int_{-\infty}^{\infty} \max _{i \in\{1,2\}} \frac{\ln \left[\left|x^{\delta} y\right|^{\gamma}+\left(x^{\delta} y\right)^{\gamma} \cos \alpha_{i}\right] f(x)}{\left[\left|x^{\delta} y\right|^{\gamma}+\left(x^{\delta} y\right)^{\gamma} \cos \alpha_{i}\right]^{\lambda / \gamma}-1} d x\right\}^{p} d y \\
& \leq K^{p}(\sigma) \int_{-\infty}^{\infty}|x|^{p(1-\delta \sigma)-1} f^{p}(x) d x .
\end{aligned}
$$

Proof For simplifying in the following, we set

$$
h^{(\delta)}(x, y):=\max _{i \in\{1,2\}} \frac{\ln \left[\left|x^{\delta} y\right|^{\gamma}+\left(x^{\delta} y\right)^{\gamma} \cos \alpha_{i}\right]}{\left[\left|x^{\delta} y\right|^{\gamma}+\left(x^{\delta} y\right)^{\gamma} \cos \alpha_{i}\right]^{\lambda / \gamma}-1} \quad(x, y \in \mathbf{R}) .
$$

By Hölder's inequality ( $c f$. [29]), we have

$$
\begin{aligned}
& \left(\int_{-\infty}^{\infty} h^{(\delta)}(x, y) f(x) d x\right)^{p} \\
& \quad=\left\{\int_{-\infty}^{\infty} h^{(\delta)}(x, y)\left[\frac{|x|^{(1-\delta \sigma) / q}}{|y|^{(1-\sigma) / p}} f(x)\right]\left[\frac{|y|^{(1-\sigma) / p}}{|x|^{(1-\delta \sigma) / q}}\right] d x\right\}^{p} \\
& \quad \leq \int_{-\infty}^{\infty} h^{(\delta)}(x, y) \frac{|x|^{(1-\delta \sigma)(p-1)}}{|y|^{1-\sigma}} f^{p}(x) d x\left[\int_{-\infty}^{\infty} h^{(\delta)}(x, y) \frac{|y|^{(1-\sigma)(q-1)}}{|x|^{1-\delta \sigma}} d x\right]^{p-1} \\
& \quad=(\omega(\sigma, y))^{p-1}|y|^{-p \sigma+1} \int_{-\infty}^{\infty} h^{(\delta)}(x, y) \frac{|x|^{(1-\delta \sigma)(p-1)}}{|y|^{1-\sigma}} f^{p}(x) d x .
\end{aligned}
$$


Then by (6) and the Fubini theorem (cf. [30]), it follows that

$$
\begin{aligned}
J & \leq K^{p-1}(\sigma) \int_{-\infty}^{\infty}\left[\int_{-\infty}^{\infty} h^{(\delta)}(x, y) \frac{|x|^{(1-\delta \sigma)(p-1)}}{|y|^{1-\sigma}} f^{p}(x) d x\right] d y \\
& =K^{p-1}(\sigma) \int_{-\infty}^{\infty} \varpi(\sigma, x)|x|^{p(1-\delta \sigma)-1} f^{p}(x) d x .
\end{aligned}
$$

Hence, still in view of (6), inequality (8) follows.

\section{Main results and applications}

Theorem 3 If $p>1, \frac{1}{p}+\frac{1}{q}=1,0<\alpha_{1} \leq \alpha_{2}<\pi, \mu, \sigma>0, \mu+\sigma=\lambda, \gamma \in\{a$; $a=$ $\left.\frac{1}{2 k+1}, 2 k-1(k \in \mathbf{N})\right\}, \delta \in\{-1,1\}, K(\sigma)$ is indicated by $(6), f(x), g(y) \geq 0$, satisfying $0<$ $\int_{-\infty}^{\infty}|x|^{p(1-\delta \sigma)-1} f^{p}(x) d x<\infty$ and $0<\int_{-\infty}^{\infty}|y|^{q(1-\sigma)-1} g^{q}(y) d y<\infty$, then we have

$$
\begin{aligned}
I & :=\int_{-\infty}^{\infty} \int_{-\infty}^{\infty} \max _{i \in\{1,2\}} \frac{\ln \left[\left|x^{\delta} y\right|^{\gamma}+\left(x^{\delta} y\right)^{\gamma} \cos \alpha_{i}\right]}{\left[\left|x^{\delta} y\right|^{\gamma}+\left(x^{\delta} y\right)^{\gamma} \cos \alpha_{i}\right]^{\lambda / \gamma}-1} f(x) g(y) d x d y \\
& <K(\sigma)\left[\int_{-\infty}^{\infty}|x|^{p(1-\delta \sigma)-1} f^{p}(x) d x\right]^{\frac{1}{p}}\left[\int_{-\infty}^{\infty}|y|^{q(1-\sigma)-1} g^{q}(y) d y\right]^{\frac{1}{q}}, \\
J & :=\int_{-\infty}^{\infty}|y|^{p \sigma-1}\left\{\int_{-\infty}^{\infty} \max _{i \in\{1,2\}} \frac{\ln \left[\left|x^{\delta} y\right|^{\gamma}+\left(x^{\delta} y\right)^{\gamma} \cos \alpha_{i}\right]}{\left[\left|x^{\delta} y\right|^{\gamma}+\left(x^{\delta} y\right)^{\gamma} \cos \alpha_{i}\right]^{\lambda / \gamma}-1} f(x) d x\right\}^{p} d y \\
& <K^{p}(\sigma) \int_{-\infty}^{\infty}|x|^{p(1-\delta \sigma)-1} f^{p}(x) d x
\end{aligned}
$$

where the constant factors $K(\sigma)$ and $K^{p}(\sigma)$ are the best possible. Inequalities (11) and (12) are equivalent.

In particular, for $\alpha_{1}=\alpha_{2}=\alpha \in(0, \pi), \gamma=1$ in (11) and (12), we find

$$
K(\sigma)=k(\sigma):=\frac{1}{2^{\sigma}}\left[\left(\sec \frac{\alpha}{2}\right)^{2 \sigma}+\left(\csc \frac{\alpha}{2}\right)^{2 \sigma}\right]\left[\frac{\pi}{\lambda \sin \left(\frac{\pi \sigma}{\lambda}\right)}\right]^{2}
$$

and the following equivalent inequalities:

$$
\begin{aligned}
& \int_{-\infty}^{\infty} \int_{-\infty}^{\infty} \frac{\ln \left(\left|x^{\delta} y\right|+x^{\delta} y \cos \alpha\right)}{\left(\left|x^{\delta} y\right|+x^{\delta} y \cos \alpha\right)^{\lambda}-1} f(x) g(y) d x d y \\
& <k(\sigma)\left[\int_{-\infty}^{\infty}|x|^{p(1-\delta \sigma)-1} f^{p}(x) d x\right]^{\frac{1}{p}}\left[\int_{-\infty}^{\infty}|y|^{q(1-\sigma)-1} g^{q}(y) d y\right]^{\frac{1}{q}} \\
& \int_{-\infty}^{\infty}|y|^{p \sigma-1}\left[\int_{-\infty}^{\infty} \frac{\ln \left(\left|x^{\delta} y\right|+x^{\delta} y \cos \alpha\right)}{\left(\left|x^{\delta} y\right|+x^{\delta} y \cos \alpha\right)^{\lambda}-1} f(x) d x\right]^{p} d y \\
& <k^{p}(\sigma) \int_{-\infty}^{\infty}|x|^{p(1-\sigma)-1} f^{p}(x) d x
\end{aligned}
$$

Proof If (10) takes the form of an equality for a $y \neq 0$, then there exist constants $A$ and $B$, such that they are not all zero, and

$$
A \frac{|x|^{(1-\delta \sigma)(p-1)}}{|y|^{1-\sigma}} f^{p}(x)=B \frac{|y|^{(1-\sigma)(q-1)}}{|x|^{1-\delta \sigma}} \text { a.e. in } \mathbf{R} \text {. }
$$


We suppose that $A \neq 0$ (otherwise $B=A=0$ ). Then it follows that

$$
|x|^{p(1-\delta \sigma)-1} f^{p}(x)=|y|^{q(1-\sigma)} \frac{B}{A|x|} \quad \text { a.e. in } \mathbf{R},
$$

which contradicts the fact that $0<\int_{-\infty}^{\infty}|x|^{p(1-\delta \sigma)-1} f^{p}(x) d x<\infty$. Hence (10) takes the form of a strict inequality. So does (8), and we have (12).

By the Hölder inequality ( $c f$. [29]), we find

$$
\begin{aligned}
I & =\int_{-\infty}^{\infty}\left(|y|^{\sigma-\frac{1}{p}} \int_{-\infty}^{\infty} h^{(\delta)}(x, y) f(x) d x\right)\left(|y|^{\frac{1}{p}-\sigma} g(y)\right) d y \\
& \leq J^{\frac{1}{p}}\left[\int_{-\infty}^{\infty}|y|^{q(1-\sigma)-1} g^{q}(y) d y\right]^{\frac{1}{q}} .
\end{aligned}
$$

Then by (12), we have (11). On the other hand, suppose that (11) is valid. Setting

$$
g(y):=|y|^{p \sigma-1}\left(\int_{-\infty}^{\infty} h^{(\delta)}(x, y) f(x) d x\right)^{p-1} \quad(y \in \mathbf{R})
$$

then it follows that $J=\int_{-\infty}^{\infty}|y|^{q(1-\sigma)-1} g^{q}(y) d y$. By (8), we have $J<\infty$. If $J=0$, then (12) is trivially true; if $0<J<\infty$, then by (11), we obtain

$$
\begin{aligned}
0 & <\int_{-\infty}^{\infty}|y|^{q(1-\sigma)-1} g^{q}(y) d y=J=I \\
& <K(\sigma)\left[\int_{-\infty}^{\infty}|x|^{p(1-\delta \sigma)-1} f^{p}(x) d x\right]^{\frac{1}{p}}\left[\int_{-\infty}^{\infty}|y|^{q(1-\sigma)-1} g^{q}(y) d y\right]^{\frac{1}{q}}<\infty, \\
J^{\frac{1}{p}} & =\left[\int_{-\infty}^{\infty}|y|^{q(1-\sigma)-1} g^{q}(y) d y\right]^{\frac{1}{p}}<K(\sigma)\left[\int_{-\infty}^{\infty}|x|^{p(1-\delta \sigma)-1} f^{p}(x) d x\right]^{\frac{1}{p}} .
\end{aligned}
$$

Hence we have (12), which is equivalent to (11).

We set $E_{\delta}:=\left\{x \in \mathbf{R} ;|x|^{\delta} \geq 1\right\}$, and $E_{\delta}^{+}:=E_{\delta} \cap \mathbf{R}_{+}=\left\{x \in \mathbf{R}_{+} ; x^{\delta} \geq 1\right\}$. For $\varepsilon>0$, we define two functions $\tilde{f}(x), \tilde{g}(y)$ as follows:

$$
\tilde{f}(x):=\left\{\begin{array}{ll}
|x|^{\delta\left(\sigma-\frac{2 \varepsilon}{p}\right)-1}, & x \in E_{\delta}, \\
0, & x \in \mathbf{R} \backslash E_{\delta},
\end{array} \quad \tilde{g}(y):= \begin{cases}0, & y \in(-\infty,-1) \cup(1, \infty), \\
|y|^{\sigma+\frac{2 \varepsilon}{q}-1}, & y \in[-1,1] .\end{cases}\right.
$$

Then we obtain

$$
\begin{aligned}
\tilde{L} & :=\left[\int_{-\infty}^{\infty}|x|^{p(1-\delta \sigma)-1} \tilde{f}^{p}(x) d x\right]^{\frac{1}{p}}\left[\int_{-\infty}^{\infty}|y|^{q(1-\sigma)-1} \tilde{g}^{q}(y) d y\right]^{\frac{1}{q}} \\
& =2\left(\int_{E_{\delta}^{+}} x^{-2 \delta \varepsilon-1} d x\right)^{\frac{1}{p}}\left(\int_{0}^{1} y^{2 \varepsilon-1} d y\right)^{\frac{1}{q}}=\frac{1}{\varepsilon} .
\end{aligned}
$$

We find

$$
I(x):=\int_{-1}^{1} \max _{i \in\{1,2\}} \frac{\ln \left[\left|x^{\delta} y\right|^{\gamma}+\left(x^{\delta} y\right)^{\gamma} \cos \alpha_{i}\right]|y|^{\sigma+\frac{2 \varepsilon}{q}-1}}{\left[\left|x^{\delta} y\right|^{\gamma}+\left(x^{\delta} y\right)^{\gamma} \cos \alpha_{i}\right]^{\lambda / \gamma}-1} d y=I(-x),
$$


and then $I(x)$ is an even function. In fact, setting $Y=-y$, we obtain

$$
\begin{aligned}
I(-x) & =\int_{-1}^{1} \max _{i \in\{1,2\}} \frac{\ln \left[\left|(-x)^{\delta} y\right|^{\gamma}+\left((-x)^{\delta} y\right)^{\gamma} \cos \alpha_{i}\right]|y|^{\sigma+\frac{2 \varepsilon}{q}-1}}{\left[\left|(-x)^{\delta} y\right|^{\gamma}+\left((-x)^{\delta} y\right)^{\gamma} \cos \alpha_{i}\right]^{\lambda / \gamma}-1} d y \\
& =\int_{-1}^{1} \max _{i \in\{1,2\}} \frac{\ln \left[\left|x^{\delta} Y\right|^{\gamma}+\left(x^{\delta} Y\right)^{\gamma} \cos \alpha_{i}\right]|Y|^{\sigma+\frac{2 \varepsilon}{q}-1}}{\left[\left|x^{\delta} Y\right|^{\gamma}+\left(x^{\delta} Y\right)^{\gamma} \cos \alpha_{i}\right]^{\lambda / \gamma}-1} d Y=I(x) .
\end{aligned}
$$

It follows that

$$
\begin{aligned}
\tilde{I} & =\int_{-\infty}^{\infty} \int_{-\infty}^{\infty} h^{(\delta)}(x, y) \tilde{f}(x) \tilde{g}(y) d x d y \\
& =\int_{E_{\delta}}|x|^{\delta\left(\sigma-\frac{2 \varepsilon}{p}\right)-1} I(x) d x=2 \int_{E_{\delta}^{+}} x^{\delta\left(\sigma-\frac{2 \varepsilon}{p}\right)-1} I(x) d x \\
\stackrel{u=x^{\delta} y}{=} & 2 \int_{E_{\delta}^{+}} x^{-2 \delta \varepsilon-1}\left[\int_{-x^{\delta}}^{x^{\delta}} \max _{i \in\{1,2\}} \frac{\ln \left(|u|^{\gamma}+u^{\gamma} \cos \alpha_{i}\right)|u|^{\sigma+\frac{2 \varepsilon}{q}-1}}{\left(|u|^{\gamma}+u^{\gamma} \cos \alpha_{i}\right)^{\lambda / \gamma}-1} d u\right] d x .
\end{aligned}
$$

Setting $v=x^{\delta}$ in the above integral, by the Fubini theorem ( $c f$. [30]), we find

$$
\begin{aligned}
& \tilde{I}=2 \int_{1}^{\infty} v^{-2 \varepsilon-1}\left[\int_{-\nu}^{v} \max _{i \in\{1,2\}} \frac{\ln \left(|u|^{\gamma}+u^{\gamma} \cos \alpha_{i}\right)|u|^{\sigma+\frac{2 \varepsilon}{q}-1}}{\left(|u|^{\gamma}+u^{\gamma} \cos \alpha_{i}\right)^{\lambda / \gamma}-1} d u\right] d \nu \\
& =2 \int_{1}^{\infty} v^{-2 \varepsilon-1}\left\{\int _ { 0 } ^ { \nu } \left[\max _{i \in\{1,2\}} \frac{\ln \left[u^{\gamma}\left(1+\cos \alpha_{i}\right)\right]}{\left[u\left(1+\cos \alpha_{i}\right)^{1 / \gamma}\right]^{\lambda}-1}\right.\right. \\
& \left.\left.+\max _{i \in\{1,2\}} \frac{\ln \left[u^{\gamma}\left(1-\cos \alpha_{i}\right)\right]}{\left[u\left(1-\cos \alpha_{i}\right)^{1 / \gamma}\right]^{\lambda}-1}\right] u^{\sigma+\frac{2 \varepsilon}{q}-1} d u\right\} d v \\
& =2 \int_{1}^{\infty} v^{-2 \varepsilon-1}\left\{\int_{0}^{\nu}\left[\frac{\ln \left[u^{\gamma}\left(1+\cos \alpha_{2}\right)\right]}{\left[u\left(1+\cos \alpha_{2}\right)^{1 / \gamma}\right]^{\lambda}-1}+\frac{\ln \left[u^{\gamma}\left(1-\cos \alpha_{1}\right)\right]}{\left[u\left(1-\cos \alpha_{1}\right)^{1 / \gamma}\right]^{\lambda}-1}\right] u^{\sigma+\frac{2 \varepsilon}{q}-1} d u\right\} d v \\
& =2 \int_{1}^{\infty} v^{-2 \varepsilon-1}\left\{\int_{0}^{1}\left[\frac{\ln \left[u^{\gamma}\left(1+\cos \alpha_{2}\right)\right]}{\left[u\left(1+\cos \alpha_{2}\right)^{1 / \gamma}\right]^{\lambda}-1}+\frac{\ln \left[u^{\gamma}\left(1-\cos \alpha_{1}\right)\right]}{\left[u\left(1-\cos \alpha_{1}\right)^{1 / \gamma}\right]^{\lambda}-1}\right] u^{\sigma+\frac{2 \varepsilon}{q}-1} d u\right\} d v \\
& +2 \int_{1}^{\infty} v^{-2 \varepsilon-1}\left\{\int _ { 1 } ^ { v } \left[\frac{\ln \left[u^{\gamma}\left(1+\cos \alpha_{2}\right)\right]}{\left[u\left(1+\cos \alpha_{2}\right)^{1 / \gamma}\right]^{\lambda}-1}\right.\right. \\
& \left.\left.+\frac{\ln \left[u^{\gamma}\left(1-\cos \alpha_{1}\right)\right]}{\left[u\left(1-\cos \alpha_{1}\right)^{1 / \gamma}\right]^{\lambda}-1}\right] u^{\sigma+\frac{2 \varepsilon}{q}-1} d u\right\} d v \\
& =\frac{1}{\varepsilon} \int_{0}^{1}\left[\frac{\ln \left[u^{\gamma}\left(1+\cos \alpha_{2}\right)\right] u^{\sigma+\frac{2 \varepsilon}{q}-1}}{\left[u\left(1+\cos \alpha_{2}\right)^{1 / \gamma}\right]^{\lambda}-1}+\frac{\ln \left[u^{\gamma}\left(1-\cos \alpha_{1}\right)\right] u^{\sigma+\frac{2 \varepsilon}{q}-1}}{\left[u\left(1-\cos \alpha_{1}\right)^{1 / \gamma}\right]^{\lambda}-1}\right] d u \\
& +2 \int_{1}^{\infty}\left(\int_{u}^{\infty} v^{-2 \varepsilon-1} d v\right) \\
& \times\left\{\frac{\ln \left[u^{\gamma}\left(1+\cos \alpha_{2}\right)\right] u^{\sigma+\frac{2 \varepsilon}{q}-1}}{\left[u\left(1+\cos \alpha_{2}\right)^{1 / \gamma}\right]^{\lambda}-1}+\frac{\ln \left[u^{\gamma}\left(1-\cos \alpha_{1}\right)\right] u^{\sigma+\frac{2 \varepsilon}{q}-1}}{\left[u\left(1-\cos \alpha_{1}\right)^{1 / \gamma}\right]^{\lambda}-1}\right\} d u \\
& =\frac{1}{\varepsilon}\left\{\int_{0}^{1}\left[\frac{\ln \left[u^{\gamma}\left(1+\cos \alpha_{2}\right)\right] u^{\sigma+\frac{2 \varepsilon}{q}-1}}{\left[u\left(1+\cos \alpha_{2}\right)^{1 / \gamma}\right]^{\lambda}-1}+\frac{\ln \left[u^{\gamma}\left(1-\cos \alpha_{1}\right)\right] u^{\sigma+\frac{2 \varepsilon}{q}-1}}{\left[u\left(1-\cos \alpha_{1}\right)^{1 / \gamma}\right]^{\lambda}-1}\right] d u\right. \\
& \left.+\int_{1}^{\infty}\left[\frac{\ln \left[u^{\gamma}\left(1+\cos \alpha_{2}\right)\right] u^{\sigma-\frac{2 \varepsilon}{p}-1}}{\left[u\left(1+\cos \alpha_{2}\right)^{1 / \gamma}\right]^{\lambda}-1}+\frac{\ln \left[u^{\gamma}\left(1-\cos \alpha_{1}\right)\right] u^{\sigma-\frac{2 \varepsilon}{p}-1}}{\left[u\left(1-\cos \alpha_{1}\right)^{1 / \gamma}\right]^{\lambda}-1}\right] d u\right\} .
\end{aligned}
$$


If the constant factor $K(\sigma)$ in (11) is not the best possible, then there exists a positive number $k$, with $K(\sigma)<k$, such that (11) is valid when replacing $K(\sigma)$ by $k$. Then we have $\varepsilon \tilde{I}<\varepsilon k \tilde{L}$, and

$$
\begin{aligned}
& \int_{0}^{1}\left\{\frac{\ln \left[u^{\gamma}\left(1+\cos \alpha_{2}\right)\right] u^{\sigma+\frac{2 \varepsilon}{q}-1}}{\left[u\left(1+\cos \alpha_{2}\right)^{1 / \gamma}\right]^{\lambda}-1}+\frac{\ln \left[u^{\gamma}\left(1-\cos \alpha_{1}\right)\right] u^{\sigma+\frac{2 \varepsilon}{q}-1}}{\left[u\left(1-\cos \alpha_{1}\right)^{1 / \gamma}\right]^{\lambda}-1}\right\} d u \\
& \quad+\int_{1}^{\infty}\left\{\frac{\ln \left[u^{\gamma}\left(1+\cos \alpha_{2}\right)\right] u^{\sigma-\frac{2 \varepsilon}{p}-1}}{\left[u\left(1+\cos \alpha_{2}\right)^{1 / \gamma}\right]^{\lambda}-1}+\frac{\ln \left[u^{\gamma}\left(1-\cos \alpha_{1}\right)\right] u^{\sigma-\frac{2 \varepsilon}{p}-1}}{\left[u\left(1-\cos \alpha_{1}\right)^{1 / \gamma}\right]^{\lambda}-1}\right\} d u \\
& =\varepsilon \tilde{I}<\varepsilon k \tilde{L}=k .
\end{aligned}
$$

By (7) and the Levi theorem ( $c f$. [30]), we have

$$
\begin{aligned}
K(\sigma)= & \int_{0}^{\infty} \frac{\ln \left[u^{\gamma}\left(1+\cos \alpha_{2}\right)\right] u^{\sigma-1} d u}{\left[u\left(1+\cos \alpha_{2}\right)^{1 / \gamma}\right]^{\lambda}-1}+\int_{0}^{\infty} \frac{\ln \left[u^{\gamma}\left(1-\cos \alpha_{1}\right)\right] u^{\sigma-1} d u}{\left[u\left(1-\cos \alpha_{1}\right)^{1 / \gamma}\right]^{\lambda}-1} \\
= & \int_{0}^{1} \lim _{\varepsilon \rightarrow 0^{+}}\left\{\frac{\ln \left[u^{\gamma}\left(1+\cos \alpha_{2}\right)\right] u^{\sigma+\frac{2 \varepsilon}{q}-1}}{\left[u\left(1+\cos \alpha_{2}\right)^{1 / \gamma}\right]^{\lambda}-1}+\frac{\ln \left[u^{\gamma}\left(1-\cos \alpha_{1}\right)\right] u^{\sigma+\frac{2 \varepsilon}{q}-1}}{\left[u\left(1-\cos \alpha_{1}\right)^{1 / \gamma}\right]^{\lambda}-1}\right\} d u \\
& +\int_{1}^{\infty} \lim _{\varepsilon \rightarrow 0^{+}}\left\{\frac{\ln \left[u^{\gamma}\left(1+\cos \alpha_{2}\right)\right] u^{\sigma-\frac{2 \varepsilon}{p}-1}}{\left[u\left(1+\cos \alpha_{2}\right)^{1 / \gamma}\right]^{\lambda}-1}+\frac{\ln \left[u^{\gamma}\left(1-\cos \alpha_{1}\right)\right] u^{\sigma-\frac{2 \varepsilon}{p}-1}}{\left[u\left(1-\cos \alpha_{1}\right)^{1 / \gamma}\right]^{\lambda}-1}\right\} d u \\
= & \lim _{\varepsilon \rightarrow 0^{+}}\left\{\int_{0}^{1}\left[\frac{\ln \left[u^{\gamma}\left(1+\cos \alpha_{2}\right)\right] u^{\sigma+\frac{2 \varepsilon}{q}-1}}{\left[u\left(1+\cos \alpha_{2}\right)^{1 / \gamma}\right]^{\lambda}-1}+\frac{\ln \left[u^{\gamma}\left(1-\cos \alpha_{1}\right)\right] u^{\sigma+\frac{2 \varepsilon}{q}-1}}{\left[u\left(1-\cos \alpha_{1}\right)^{1 / \gamma}\right]^{\lambda}-1}\right] d u\right. \\
& \left.+\int_{1}^{\infty}\left[\frac{\ln \left[u^{\gamma}\left(1+\cos \alpha_{2}\right)\right] u^{\sigma-\frac{2 \varepsilon}{p}-1}}{\left[u\left(1+\cos \alpha_{2}\right)^{1 / \gamma}\right]^{\lambda}-1}+\frac{\ln \left[u^{\gamma}\left(1-\cos \alpha_{1}\right)\right] u^{\sigma-\frac{2 \varepsilon}{p}-1}}{\left[u\left(1-\cos \alpha_{1}\right)^{1 / \gamma}\right]^{\lambda}-1}\right] d u\right\} \leq k,
\end{aligned}
$$

which contradicts the fact that $k<K(\sigma)$. Hence the constant factor $K(\sigma)$ in $(11)$ is the best possible.

If the constant factor in (12) is not the best possible, then by (16), we would reach a contradiction: that the constant factor in (11) is not the best possible.

Theorem 4 On the assumptions of Theorem 3, replacing $p>1$ by $0<p<1$, we have the equivalent reverses of (11) and (12) with the same best constant factors.

Proof By the reverse Hölder inequality ( $c f$. [29]), we have the reverses of (9) and (16). It is easy to obtain the reverse of (12). In view of the reverses of (12) and (16), we obtain the reverse of (11). On the other hand, suppose that the reverse of (11) is valid. Setting the same $g(y)$ as Theorem 3, by the reverse of (9), we have $J>0$. If $J=\infty$, then the reverse of (12) is trivially value; if $J<\infty$, then by the reverse of (11), we obtain the reverses of (17) and (18). Hence we have the reverse of (12), which is equivalent to the reverse of (11).

If the constant factor $K(\sigma)$ in the reverse of (11) is not the best possible, then there exists a positive constant $k$, with $k>K(\sigma)$, such that the reverse of (11) is still valid when replacing $K(\sigma)$ by $k$. By the reverse of (19), we have

$$
\begin{aligned}
& \int_{0}^{1}\left\{\frac{\ln \left[u^{\gamma}\left(1+\cos \alpha_{2}\right)\right] u^{\sigma+\frac{2 \varepsilon}{q}-1}}{\left[u\left(1+\cos \alpha_{2}\right)^{1 / \gamma}\right]^{\lambda}-1}+\frac{\ln \left[u^{\gamma}\left(1-\cos \alpha_{1}\right)\right] u^{\sigma+\frac{2 \varepsilon}{q}-1}}{\left[u\left(1-\cos \alpha_{1}\right)^{1 / \gamma}\right]^{\lambda}-1}\right\} d u \\
& \quad+\int_{1}^{\infty}\left\{\frac{\ln \left[u^{\gamma}\left(1+\cos \alpha_{2}\right)\right] u^{\sigma-\frac{2 \varepsilon}{p}-1}}{\left[u\left(1+\cos \alpha_{2}\right)^{1 / \gamma}\right]^{\lambda}-1}+\frac{\ln \left[u^{\gamma}\left(1-\cos \alpha_{1}\right)\right] u^{\sigma-\frac{2 \varepsilon}{p}-1}}{\left[u\left(1-\cos \alpha_{1}\right)^{1 / \gamma}\right]^{\lambda}-1}\right\} d u>k .
\end{aligned}
$$


For $\varepsilon \rightarrow 0^{+}$, by the Levi theorem (cf. [30]), we find

$$
\begin{aligned}
\int_{1}^{\infty} & \left\{\frac{\ln \left[u^{\gamma}\left(1+\cos \alpha_{2}\right)\right] u^{\sigma-\frac{2 \varepsilon}{p}-1}}{\left[u\left(1+\cos \alpha_{2}\right)^{1 / \gamma}\right]^{\lambda}-1}+\frac{\ln \left[u^{\gamma}\left(1-\cos \alpha_{1}\right)\right] u^{\sigma-\frac{2 \varepsilon}{p}-1}}{\left[u\left(1-\cos \alpha_{1}\right)^{1 / \gamma}\right]^{\lambda}-1}\right\} d u \\
& \rightarrow \int_{1}^{\infty}\left\{\frac{\ln \left[u^{\gamma}\left(1+\cos \alpha_{2}\right)\right] u^{\sigma-1}}{\left[u\left(1+\cos \alpha_{2}\right)^{1 / \gamma}\right]^{\lambda}-1}+\frac{\ln \left[u^{\gamma}\left(1-\cos \alpha_{1}\right)\right] u^{\sigma-1}}{\left[u\left(1-\cos \alpha_{1}\right)^{1 / \gamma}\right]^{\lambda}-1}\right\} d u
\end{aligned}
$$

There exists a constant $\delta_{0}>0$, such that $\sigma-\frac{1}{2} \delta_{0}>0$, and then $K\left(\sigma-\frac{\delta_{0}}{2}\right) \in \mathbf{R}_{+}$. For $0<$ $\varepsilon<\frac{\delta_{0}|q|}{4}(q<0)$, since $u^{\sigma+\frac{2 \varepsilon}{q}-1} \leq u^{\sigma-\frac{\delta_{0}}{2}-1}, u \in(0,1]$, and

$$
\begin{aligned}
0 & <\int_{0}^{1}\left\{\frac{\ln \left[u^{\gamma}\left(1+\cos \alpha_{2}\right)\right] u^{\sigma-\frac{\delta_{0}}{2}-1}}{\left[u\left(1+\cos \alpha_{2}\right)^{1 / \gamma}\right]^{\lambda}-1}+\frac{\ln \left[u^{\gamma}\left(1-\cos \alpha_{1}\right)\right] u^{\sigma-\frac{\delta_{0}}{2}-1}}{\left[u\left(1-\cos \alpha_{1}\right)^{1 / \gamma}\right]^{\lambda}-1}\right\} d u \\
& \leq K\left(\sigma-\frac{\delta_{0}}{2}\right),
\end{aligned}
$$

then by the Lebesgue control convergence theorem (cf. [30]), for $\varepsilon \rightarrow 0^{+}$, we have

$$
\begin{aligned}
& \int_{0}^{1}\left\{\frac{\ln \left[u^{\gamma}\left(1+\cos \alpha_{2}\right)\right] u^{\sigma+\frac{2 \varepsilon}{q}-1}}{\left[u\left(1+\cos \alpha_{2}\right)^{1 / \gamma}\right]^{\lambda}-1}+\frac{\ln \left[u^{\gamma}\left(1-\cos \alpha_{1}\right)\right] u^{\sigma+\frac{2 \varepsilon}{q}-1}}{\left[u\left(1-\cos \alpha_{1}\right)^{1 / \gamma}\right]^{\lambda}-1}\right\} d u \\
& \rightarrow \int_{0}^{1}\left\{\frac{\ln \left[u^{\gamma}\left(1+\cos \alpha_{2}\right)\right] u^{\sigma-1}}{\left[u\left(1+\cos \alpha_{2}\right)^{1 / \gamma}\right]^{\lambda}-1}+\frac{\ln \left[u^{\gamma}\left(1-\cos \alpha_{1}\right)\right] u^{\sigma-1}}{\left[u\left(1-\cos \alpha_{1}\right)^{1 / \gamma}\right]^{\lambda}-1}\right\} d u
\end{aligned}
$$

By (20), (21), and (22), for $\varepsilon \rightarrow 0^{+}$, we find $K(\sigma) \geq k$, which contradicts the fact that $k>$ $K(\sigma)$. Hence, the constant factor $K(\sigma)$ in the reverse of (11) is the best possible.

If the constant factor in reverse of (12) is not the best possible, then by the reverse of (16), we would reach a contradiction: that the constant factor in the reverse of (11) is not the best possible.

Corollary 5 For $\delta=-1$ in (11) and (12), replacing $|x|^{\lambda} f(x)$ by $f(x)$, we obtain

$$
0<\int_{-\infty}^{\infty}|x|^{p(1-\mu)-1} f^{p}(x) d x<\infty
$$

and the following equivalent inequalities with the homogeneous kernel and the best possible constant factors:

$$
\begin{aligned}
& \int_{-\infty}^{\infty} \int_{-\infty}^{\infty} \max _{i \in\{1,2\}} \frac{\ln \left[\left(|y|^{\gamma}+\operatorname{sgn}(x) y^{\gamma} \cos \alpha_{i}\right) /|x|^{\gamma}\right]}{\left[|y|^{\gamma}+\operatorname{sgn}(x) y^{\gamma} \cos \alpha_{i}\right]^{\lambda / \gamma}-|x|^{\lambda}} f(x) g(y) d x d y \\
& <K(\sigma)\left[\int_{-\infty}^{\infty}|x|^{p(1-\mu)-1} f^{p}(x) d x\right]^{\frac{1}{p}}\left[\int_{-\infty}^{\infty}|y|^{q(1-\sigma)-1} g^{q}(y) d y\right]^{\frac{1}{q}}, \\
& \int_{-\infty}^{\infty}|y|^{p \sigma-1}\left\{\int_{-\infty}^{\infty} \max _{i \in\{1,2\}} \frac{\ln \left[\left(|y|^{\gamma}+\operatorname{sgn}(x) y^{\gamma} \cos \alpha_{i}\right) /|x|^{\gamma}\right]}{\left[|y|^{\gamma}+\operatorname{sgn}(x) y^{\gamma} \cos \alpha_{i}\right]^{\lambda / \gamma}-|x|^{\lambda}} f(x) d x\right\}^{p} d y \\
& <K^{p}(\sigma) \int_{-\infty}^{\infty}|x|^{p(1-\mu)-1} f^{p}(x) d x .
\end{aligned}
$$

In particular, for $\alpha_{1}=\alpha_{2}=\alpha \in(0, \pi), \gamma=1$ in (23) and (24), we obtain the following equivalent inequalities: 


$$
\begin{aligned}
& \int_{-\infty}^{\infty} \int_{-\infty}^{\infty} \frac{\ln [(|y|+\operatorname{sgn}(x) y \cos \alpha) /|x|]}{[|y|+\operatorname{sgn}(x) y \cos \alpha]^{\lambda}-|x|^{\lambda}} f(x) g(y) d x d y \\
& <k(\sigma)\left[\int_{-\infty}^{\infty}|x|^{p(1-\mu)-1} f^{p}(x) d x\right]^{\frac{1}{p}}\left[\int_{-\infty}^{\infty}|y|^{q(1-\sigma)-1} g^{q}(y) d y\right]^{\frac{1}{q}}, \\
& \int_{-\infty}^{\infty}|y|^{p \sigma-1}\left\{\int_{-\infty}^{\infty} \frac{\ln [(|y|+\operatorname{sgn}(x) y \cos \alpha) /|x|]}{[|y|+\operatorname{sgn}(x) y \cos \alpha]^{\lambda}-|x|^{\lambda}} f(x) d x\right\}^{p} d y \\
& <k^{p}(\sigma) \int_{-\infty}^{\infty}|x|^{p(1-\mu)-1} f^{p}(x) d x,
\end{aligned}
$$

where $k(\sigma)$ is indicated by (13).

\section{Two kinds of operator expressions}

Suppose that $p>1, \frac{1}{p}+\frac{1}{q}=1,0<\alpha_{1} \leq \alpha_{2}<\pi, \mu, \sigma>0, \mu+\sigma=\lambda, \gamma \in\left\{a ; a=\frac{1}{2 k+1}, 2 k-1(k \in\right.$ N) $\}, \delta \in\{-1,1\}$. We set the following functions:

$$
\varphi(x):=|x|^{p(1-\delta \sigma)-1}, \quad \psi(y):=|y|^{q(1-\sigma)-1}, \quad \phi(x):=|x|^{p(1-\mu)-1} \quad(x, y \in \mathbf{R}),
$$

therefore, $\psi^{1-p}(y)=|y|^{p \sigma-1}$. Define the following real normed linear space:

$$
\begin{aligned}
& L_{p, \varphi}(\mathbf{R}):=\left\{f:\|f\|_{p, \varphi}:=\left(\int_{-\infty}^{\infty} \varphi(x)|f(x)|^{p} d x\right)^{\frac{1}{p}}<\infty\right\}, \\
& L_{p, \psi^{1}-p}(\mathbf{R})=\left\{h:\|h\|_{p, \psi^{1-p}}=\left(\int_{-\infty}^{\infty} \psi^{1-p}(y)|h(y)|^{p} d y\right)^{\frac{1}{p}}<\infty\right\}, \\
& L_{p, \phi}(\mathbf{R})=\left\{g:\|g\|_{p, \phi}=\left(\int_{-\infty}^{\infty} \phi(x)|g(x)|^{p} d x\right)^{\frac{1}{p}}<\infty\right\} .
\end{aligned}
$$

(a) In view of Theorem 3 , for $f \in L_{p, \varphi}(\mathbf{R})$, setting

$$
H_{1}(y):=\int_{-\infty}^{\infty} \max _{i \in\{1,2\}} \frac{\ln \left[\left|x^{\delta} y\right|^{\gamma}+\left(x^{\delta} y\right)^{\gamma} \cos \alpha_{i}\right]}{\left[\left|x^{\delta} y\right|^{\gamma}+\left(x^{\delta} y\right)^{\gamma} \cos \alpha_{i}\right]^{\lambda / \gamma}-1} f(x) d x \quad(y \in \mathbf{R}),
$$

by (12), we have

$$
\left\|H_{1}\right\|_{p, \psi^{1-p}}=\left(\int_{-\infty}^{\infty} \psi^{1-p}(y) H_{1}^{p}(y) d y\right)^{\frac{1}{p}}<K(\sigma)\|f\|_{p, \varphi}<\infty
$$

Definition 1 Define a Hilbert-type integral operator with the non-homogeneous kernel in the whole plane $T_{1}: L_{p, \varphi}(\mathbf{R}) \rightarrow L_{p, \psi^{1-p}}(\mathbf{R})$ as follows: For any $f \in L_{p, \varphi}(\mathbf{R})$, there exists a unique representation $T_{1} f=H_{1} \in L_{p, \psi^{1-p}}(\mathbf{R})$, satisfying, for any $y \in \mathbf{R}, T_{1} f(y)=H_{1}(y)$.

In view of (27), it follows that $\left\|T_{1} f\right\|_{p, \psi^{1-p}}=\left\|H_{1}\right\|_{p, \psi^{1-p}} \leq K(\sigma)\|f\|_{p, \varphi}$, and then the operator $T_{1}$ is bounded satisfying

$$
\left\|T_{1}\right\|=\sup _{f(\not \theta) \in L_{p, \varphi}(\mathbf{R})} \frac{\left\|T_{1} f\right\|_{p, \psi}{ }^{1-p}}{\|f\|_{p, \varphi}} \leq K(\sigma) .
$$

Since the constant factor $K(\sigma)$ in (27) is the best possible, we have $\left\|T_{1}\right\|=K(\sigma)$. 
If we define the formal inner product of $T_{1} f$ and $g$ as

$$
\begin{aligned}
\left(T_{1} f, g\right) & :=\int_{-\infty}^{\infty}\left(\int_{-\infty}^{\infty} h^{(\delta)}(x, y) f(x) d x\right) g(y) d y \\
& =\int_{-\infty}^{\infty} \int_{-\infty}^{\infty} h^{(\delta)}(x, y) f(x) g(y) d x d y
\end{aligned}
$$

then we can rewrite (11) and (12) as follows:

$$
\left(T_{1} f, g\right)<\left\|T_{1}\right\| \cdot\|f\|_{p, \varphi}\|g\|_{q, \psi}, \quad\left\|T_{1} f\right\|_{p, \psi^{1-p}}<\left\|T_{1}\right\| \cdot\|f\|_{p, \varphi} .
$$

(b) In view of Corollary 5 , for $f \in L_{p, \phi}(\mathbf{R})$, setting

$$
H_{2}(y):=\int_{-\infty}^{\infty} \max _{i \in\{1,2\}} \frac{\ln \left[\left(|y|^{\gamma}+\operatorname{sgn}(x) y^{\gamma} \cos \alpha_{i}\right) /|x|^{\gamma}\right]}{\left[|y|^{\gamma}+\operatorname{sgn}(x) y^{\gamma} \cos \alpha_{i}\right]^{\lambda / \gamma}-|x|^{\lambda}} f(x) d x \quad(y \in \mathbf{R}),
$$

by (24), we have

$$
\left\|H_{2}\right\|_{p, \psi^{1-p}}=\left(\int_{-\infty}^{\infty} \psi^{1-p}(y) H_{2}^{p}(y) d y\right)^{\frac{1}{p}}<K(\sigma)\|f\|_{p, \phi}<\infty
$$

Definition 2 Define a Hilbert-type integral operator with the homogeneous kernel in the whole plane $T_{2}: L_{p, \phi}(\mathbf{R}) \rightarrow L_{p, \psi^{1-p}}(\mathbf{R})$ as follows: For any $f \in L_{p, \phi}(\mathbf{R})$, there exists a unique representation $T_{2} f=H_{2} \in L_{p, \psi^{1-p}}(\mathbf{R})$, satisfying, for any $y \in \mathbf{R}, T_{2} f(y)=H_{2}(y)$.

In view of (28), it follows that $\left\|T_{2} f\right\|_{p, \psi^{1-p}}=\left\|H_{2}\right\|_{p, \psi^{1-p}} \leq K(\sigma)\|f\|_{p, \phi}$, and then the operator $T_{2}$ is bounded satisfying

$$
\left\|T_{2}\right\|=\sup _{f(\neq \theta) \in L_{p, \phi}(\mathbf{R})} \frac{\left\|T_{2} f\right\|_{p, \psi^{1-p}}}{\|f\|_{p, \phi}} \leq K(\sigma) .
$$

Since the constant factor $K(\sigma)$ in (28) is the best possible, we have $\left\|T_{2}\right\|=K(\sigma)$.

If we define the formal inner product of $T_{2} f$ and $g$ as

$$
\left(T_{2} f, g\right):=\int_{-\infty}^{\infty} \int_{-\infty}^{\infty} \max _{i \in\{1,2\}} \frac{\ln \left[\left(|y|^{\gamma}+\operatorname{sgn}(x) y^{\gamma} \cos \alpha_{i}\right) /|x|^{\gamma}\right]}{\left[|y|^{\gamma}+\operatorname{sgn}(x) y^{\gamma} \cos \alpha_{i}\right]^{\lambda / \gamma}-|x|^{\lambda}} f(x) g(y) d x d y
$$

then we can rewrite (23) and (24) as follows:

$$
\left(T_{2} f, g\right)<\left\|T_{2}\right\| \cdot\|f\|_{p, \phi}\|g\|_{q, \psi}, \quad\left\|T_{2} f\right\|_{p, \psi 1-p}<\left\|T_{2}\right\| \cdot\|f\|_{p, \phi} .
$$

\section{Competing interests}

The authors declare that they have no competing interests.

\section{Authors' contributions}

BY carried out the mathematical studies, participated in the sequence alignment and drafted the manuscript. QC participated in the design of the study and performed the numerical analysis. All authors read and approved the final manuscript.

\section{Author details}

'Department of Mathematics, Guangdong University of Education, Guangzhou, Guangdong 510303, P.R. China.

2Department of Computer Science, Guangdong University of Education, Guangzhou, Guangdong 510303, P.R. China. 


\section{Acknowledgements}

This work is supported by the National Natural Science Foundation of China (No. 61370186), and 2013 Knowledge Construction Special Foundation Item of Guangdong Institution of Higher Learning College and University (No. 2013KJCX0140)

Received: 23 August 2014 Accepted: 24 December 2014 Published online: 22 January 2015

\section{References}

1. Hardy, GH, Littlewood, JE, Pólya, G: Inequalities. Cambridge University Press, Cambridge (1934)

2. Mitrinović, DS, Pecaric, JE, Fink, AM: Inequalities Involving Functions and Their Integrals and Derivatives. Kluwer Academic, Boston (1991)

3. Yang, BC: The Norm of Operator and Hilbert-Type Inequalities. Science Press, Beijing (2009)

4. Yang, BC: A survey of the study of Hilbert-type inequalities with parameters. Adv. Math. 38(3), 257-268 (2009)

5. Yang, BC: On the norm of an integral operator and applications. J. Math. Anal. Appl. 321, 182-192 (2006)

6. Xu, JS: Hardy-Hilbert's inequalities with two parameters. Adv. Math. 36(2), 63-76 (2007)

7. Yang, BC: On the norm of a Hilbert's type linear operator and applications. J. Math. Anal. Appl. 325, 529-541 (2007)

8. Xin, DM: A Hilbert-type integral inequality with the homogeneous kernel of zero degree. Math. Theory Appl. 30(2), 70-74 (2010)

9. Yang, BC: A Hilbert-type integral inequality with the homogeneous kernel of degree 0. J. Shandong Univ. Nat. Sci. 45(2), 103-106 (2010)

10. Debnath, L, Yang, BC: Recent developments of Hilbert-type discrete and integral inequalities with applications. Int. J. Math. Math. Sci. 2012, Article ID 871845 (2012)

11. Rassias, MT, Yang, BC: On half-discrete Hilbert's inequality. Appl. Math. Comput. 220, 75-93 (2013)

12. Yang, BC, Krnic, M: A half-discrete Hilbert-type inequality with a general homogeneous kernel of degree 0 . J. Math. Inequal. 6(3), 401-417 (2012)

13. Rassias, MT, Yang, BC: A multidimensional half-discrete Hilbert-type inequality and the Riemann zeta function. Appl. Math. Comput. 225, 263-277 (2013)

14. Rassias, MT, Yang, BC: On a multidimensional half - discrete Hilbert - type inequality related to the hyperbolic cotangent function. Appl. Math. Comput. 242, 800-813 (2013)

15. Rassias, MT, Yang, BC: A multidimensional Hilbert-type integral inequality related to the Riemann zeta function. In: Daras, NJ (ed.) Applications of Mathematics and Informatics in Science and Engineering, pp. 417-433. Springer, New York (2014)

16. Yang, BC: A new Hilbert-type integral inequality. Soochow J. Math. 33(4), 849-859 (2007)

17. Wang, ZQ, Guo, DR: Introduction to Special Functions. Science Press, Beijing (1979)

18. He, B, Yang, BC: On a Hilbert-type integral inequality with the homogeneous kernel of 0-degree and the hypergeometric function. Math. Pract. Theory 40(18), 105-211 (2010)

19. Yang, BC: A new Hilbert-type integral inequality with some parameters. J. Jilin Univ. Sci. Ed. 46(6), 1085-1090 (2008)

20. Yang, BC: A Hilbert-type integral inequality with a non-homogeneous kernel. J. Xiamen Univ. Natur. Sci. 48(2), 165-169 (2008)

21. Zeng, Z, Xie, ZT: On a new Hilbert-type integral inequality with the homogeneous kernel of degree 0 and the integral in whole plane. J. Inequal. Appl. 2010, Article ID 256796 (2010)

22. Yang, BC: A reverse Hilbert-type integral inequality with some parameters. J. Xinxiang Univ. Natur. Sci. Ed. 27(6), 1-4 (2010)

23. Wang, AZ, Yang, BC: A new Hilbert-type integral inequality in whole plane with the non-homogeneous kernel. J. Inequal. Appl. 2011, Article ID 123 (2011). doi:10.1186/1029-24X-2011-123

24. Xin, DM, Yang, BC: A Hilbert-type integral inequality in whole plane with the homogeneous kernel of degree -2 . J. Inequal. Appl. 2011, Article ID 401428 (2011)

25. He, B, Yang, BC: On an inequality concerning a non-homogeneous kernel and the hypergeometric function. Tamsui Oxf. J. Inf. Math. Sci. 27(1), 75-88 (2011)

26. Yang, BC: A reverse Hilbert-type integral inequality with a non-homogeneous kernel. J. Jilin Univ. Sci. Ed. 49(3), 437-441 (2011)

27. Xie, ZT, Zeng, Z, Sun, YF: A new Hilbert-type inequality with the homogeneous kernel of degree -2. Adv. Appl. Math. Sci. 12(7), 391-401 (2013)

28. Zeng, Z, Raja Rama Gandhi, K, Xie, ZT: A new Hilbert-type inequality with the homogeneous kernel of degree -2 and with the integral. Bull. Math. Sci. Appl. 3(1), 11-20 (2014)

29. Kuang, J: Applied Inequalities. Shangdong Science and Technology Press, Jinan (2004)

30. Kuang, J: Introduction to Real Analysis. Hunan Education Press, Changsha (1996) 